\title{
Prevalencia de alteraciones musculoesqueléticas en el pie infantil: estudio preliminar
}

\author{
Prevalence of musculoskeletal disorders in children's foot: \\ preliminary study
}

Laura Cala Pérez ${ }^{1}$, Marta Elena Losa Iglesias ${ }^{2}$

${ }^{1}$ Diplomada en Podología. Máster de Investigación en Podología. Universidad Rey Juan Carlos de Madrid. laura.cala@hotmail.com

${ }^{2}$ Diplomada en Podología. Doctora Por la Universidad de Oviedo. Directora Máster de Investigación en Podología. Universidad Rey Juan Carlos de Madrid. marta.losa@urjc.es

Correspondencia:

Laura Cala Pérez

Podólogos Cala S.L.P.

c/ Rey Pelayo $n^{\circ} 28$, Piedras Blancas, Asturias.

Correo electrónico: laura.cala@hotmail.com; marta.losa@urjc.es

Fecha de recepción: 20 de mayo de 2013

Fecha de aceptación: 22 de julio de 2013

Los autores declaran no tener ningún tipo de interés económico o comercial.

\section{RESUMEN}

A la edad escolar ya se tiene la marcha, la funcionalidad y la morfología del pie característica del adulto pero aún no está completada la madurez esquelética. Esto hace que sea una edad propicia para realizar una adecuada prevención y un diagnóstico precoz de las alteraciones musculoesqueléticas de los pies y miembros inferiores. Hemos realizado un estudio transversal de prevalencia para conocer el estado de salud de los pies de los escolares. Se han evaluado 116 niños mediante una observación clínica y un estudio de la marcha con una plataforma de presiones. Los resultados más llamativos fueron que al 93\% nunca se les había realizado una exploración similar, el 95\% tenía alterado el reparto de presiones de la huella, un 75\% presentaba alguna alteración en la dinámica (la más frecuente excesiva pronación en la fase de propulsión) y el mismo porcentaje de niños llevaban el calzado de talla pequeña. Más de la mitad tenía una huella anormal y el pie excesivamente pronado. Un $40 \%$ presentaba dolor, el $35 \%$ tenía pie cavo y un $10 \%$ pie plano. El 32\% tenía alguna patología de antepie. El resto de alteraciones fueron menos frecuentes. Hemos encontrado una alta prevalencia de alteraciones, por lo que se evidencia una vez más la necesidad de hacer controles periódicos a estas edades e incluirlos dentro de los programas de educación para la salud y prevenir futuras complicaciones.

Palabras clave: alteraciones musculoesqueléticas; patologías pies; edad escolar; prevalencia.

\begin{abstract}
At school age, children already have the functionality and foot morphology of adult but have not yet completed skeletal maturity. This makes it a proper age for appropriate prevention and early diagnosis of musculoskeletal disorders of the feet and lower limbs. We conducted a cross-sectional study of prevalence for the health of the feet of the schoolaged children. 116 children have been assessed by clinical observation and a study of gait with a pressure platform. The most striking results were that $93 \%$ never had made a similar exploration, $95 \%$ had altered pressure distribution footprint, $75 \%$ showed some alteration in the dynamics (the most frequent excessive pronation phase propulsion) and the same percentage of children wearing shoes small size. More than half had an abnormal trace excessively pronated foot. Meanwhile, $40 \%$ had pain, $35 \%$ had pes cavus and flatfoot $10 \%$. Finally, $32 \%$ had one forefoot pathology. The remaining changes were less frequent. We found a high prevalence of alterations so it is further evidence of the need for regular checks at this age to prevent complications and implement programs of health education.
\end{abstract}

Keywords: musculoskeletal disorders; foot deformities; school-aged children; prevalence.

Sumario: 1. Introducción. 2. Material y Métodos. 3. Resultados. 4. Discusión. 5. Conclusiones. Bibliografía.

Referencia normalizada: Cala Pérez, L., Losa Iglesias, M.E. Prevalencia de alteraciones muscoloesqueléticas en el pie infantil: estudio preliminar. Rev. Int. Cienc. Podol. 2015; 9(1): 1-16. 


\section{INTRODUCCIÓN}

El desarrollo del pie y del miembro inferior comienza en el periodo embrionario y va sufriendo una serie de variaciones fisiológicas a lo largo del crecimiento hasta que alcanza hacia los 7-8 años, la morfología, la funcionalidad normal y la marcha característica del adulto, a pesar de que la madurez no se completa hasta el final de la adolescencia ${ }^{1,4}$. También a esta edad se corrigen de forma espontánea algunas alteraciones rotacionales de los miembros inferiores ${ }^{1,2,3}$, siendo un periodo propicio para hacer una valoración biomecánica y comprobar la correcta evolución del desarrollo.

Aunque no es fácil catalogar un pie como "normal" 4,5 , una de las clasificaciones internacionalmente validada es utilizar los criterios biofísicos de normalidad y anormalidad descritos por Merlon L. Root et al. ${ }^{4}$ que define las siguientes relaciones físicas como ideales en una posición de bipedestación estática:

- Las piernas y la bisección sagital del calcáneo son perpendiculares al suelo y paralelas la una a la otra. La articulación subtalar está colocada en posición neutra. La rodilla, tobillo y articulación subastragalina discurren en planos transversos paralelos a la superficie que los soporta.

- La articulación mediotarsiana se bloquea en su posición de máxima pronación. Los planos inferiores del antepie y retropie son paralelos entre sí y también paralelos a la superficie sobre la que se apoyan. En esta posición la bisección sagital de la superficie posterior del calcáneo es perpendicular al plano plantar del pie.

- Los metatarsianos II, III y IV se encuentran en posición de total flexión dorsal; la superficie plantar de las cabezas metatarsianas describe un plano común paralelo a la superficie de apoyo. La superficie plantar de todas las cabezas metatarsianas discurren en el mismo plano transverso.

Respecto a la dinámica, $\operatorname{Root}^{4}$ define la amplitud mínima necesaria que debe tener cada articulación del pie para una marcha normal, puesto que el movimiento limitado, una pronación o una supinación anormales, causan una marcha patológica y por consecuencia, algunas alteraciones funcionales del pie.

También es importante determinar las alteraciones en la huella y de la fórmula metatarsal de los niños buscando la asociación con algunas patologías presentes que se puedan producir por mecanismos compensatorios tal y como describe Martín Rueda, que afirma que las alteraciones morfológicas de la fórmula metatarsal van a provocar, desde la niñez, cambios del ángulo de torsión de la pierna y de la posición del antepie, para corregir la trayectoria de los vectores de carga durante la marcha 5 .

Por otro lado, la utilización de un calzado fisiológico y en talla, es otro de los puntos importantes a valorar en un pie infantil ya que es un factor extrínseco en el desarrollo de deformidades del antepie ${ }^{6,7,8,9}$. La utilización de un calzado incorrecto puede ser causa de alteraciones de la marcha o puede agravar problemas biomecánicos o morfo-funcionales que ya existían previamente. Durante esta etapa, en la que comienzan a participar en actividades deportivas, el calzado adquiere un papel fundamental y por lo tanto debe tener unas características adecuadas ${ }^{10,11,12,13,14}$.

Por todo lo anteriormente expuesto, entendemos importante investigar más sobre la patología del pie infantil, por lo que el objetivo principal de este estudio será determinar la prevalencia de patologías músculo-esqueléticas en los pies de los niños de 6 a 12 años y detectar el porcentaje de escolares que utilizan un calzado de un tamaño inadecuado.

\section{MATERIAL Y MÉTODOS}

Se realizó un estudio observacional transversal para conocer la prevalencia de patología musculoesquelética en los pies de los escolares.

Se procedió a la elaboración de una hoja informativa y la autorización de inclusión en el estudio, que previa autorización del consejo escolar del centro, se repartió a los todos los alumnos de 6 a 12 años matriculados en el colegio público de Salinas durante el curso académico 2012-2013, para su entrega a los padres.

\section{Criterios de inclusión y exclusión:}

Se han incluido en este estudio a todos los alumnos que han querido participar presen- 
tando la autorización firmada por los padres. Como único criterio de exclusión, los alumnos cuyos padres no firmaron la autorización.

\section{Duración del estudio tiempo y lugar:}

La valoración ortopédica de los niños se realizó en un aula del colegio, en horario de mañanas de $9.30 \mathrm{~h}$ a $14.00 \mathrm{~h}$. durante el horario escolar a lo largo de 18 días laborables consecutivos durante los meses de enero y febrero de 2013. Todos los niños fueron evaluados por el mismo examinador, con el fin de reducir los errores de medición. Los materiales que se han utilizado fueron una camilla, báscula con tallímetro, podoscopio, plataforma de presiones Footwork de 6.024 sensores y el software Footwork Pro 3.0.1.1, regla de Perthes, pelvímetro, goniómetro de Moltgen, plomada, cinta métrica y lápiz dermográfico.

\section{Definición de las variables:}

El registro de las variables se inicia con una evaluación antropométrica que incluía edad, sexo, peso y talla.

Comenzamos con una exploración en el podoscopio en bipedestación en carga relajada con las rodillas extendidas y ambos talones apoyados. Palpamos las espinas y las crestas iliacas anterosuperiores y valoramos con el pelvímetro la existencia de báscula pélvica. En los casos que se encontró un desnivel, se comprobó, como explicaré más adelante, con la medición en camilla ${ }^{15}$. Se valoró en esta misma posición las variaciones angulares de los miembros inferiores. Para ello se alinean los pies hasta que las rótulas miren al frente, juntando las extremidades hasta que contactan los cóndilos femorales internos o maléolos tibiales sin que se superpongan las estructuras, con el apoyo de los miembros en total normalidad sin posturas forzadas. Para la detección del genu valgo se mide con un calibre la distancia intercondílea o intermaleolar en centímetros. Se catalogó como genu valgo leve la existencia de una distancia intermaleolar de 5 a $7,5 \mathrm{~cm}$ y genu valgo marcado cuando la distancia intermaleolar supera los 7,5 cm. Por otro lado, cualquier grado de genu varo es patológico, por tanto se evidenció la alteración si juntando los maleolos con las rodillas extendidas quedan separados los cón- dilos femorales ${ }^{11,16,17,18,19}$. Para la detección de una rotación interna o externa de las rótulas se debe realizar la exploración en bipedestación con los pies juntos y paralelos. Para detectar estas deformaciones se localizó clínicamente la espina iliaca anterosuperior para trazar el eje longitudinal de la extremidad inferior hasta el centro del tobillo con la plomada. Esta línea en condiciones normales debe pasar por el centro de la rótula. A los escolares que presentaron las rótulas mirando hacia dentro respecto a esta línea, se catalogó como rótulas convergentes y hacia fuera, divergentes ${ }^{15}$.

Se observó la presencia de deformidades de los dedos: dedos en varo o aducción, en valgo o abducción, deformidad en martillo o en garra y hallux en aducción o abducción.

Se valoró la posición del pie utilizando el Foot Posture Index, cuyo uso permite cuantificar el grado de pronación, supinación o neutralidad partir de la palpación de la cabeza del astrágalo, las curvas supra e inframaleolares laterales, la posición del calcáneo, la prominencia talonavicular, la congruencia del arco longitudinal interno, y la abducción o aducción del antepié respecto al retropié. Se anotaron las posibles posturas como neutro, pronado, muy pronado, supinado y muy supinado, según los valores obtenidos observando el pie del alumno en apoyo bipodal relajado mirando al frente ${ }^{20}$.

A pesar de que la medición de los grados de inversión y eversión del calcáneo respecto al suelo no es necesario para definir esta variable, se cuantificarán igualmente debido a la cantidad de autores que consideran un retropié patológico aquel que presenta más de $5^{\circ}$ de valgo en carga relajada ${ }^{11,15,18,21,22}$ y así buscar alguna asociación de las desviaciones de retropié encontradas con otras patologías o alteraciones de la dinámica.

El tipo de huella se representó con las letras de la A a la $\mathrm{G}^{5}$. Siendo la tipo A, una huella de perímetros normales. La de tipo $\mathrm{B}$, aquella que presenta una pérdida de presión del arco externo se suele dar en el pie valgo y cavo, correspondiéndose con la tradicionalmente llamada huella cava de primer grado, pero que al corregir el valgo se normaliza dando imagen de una huella tipo A. La huella de tipo $\mathrm{C}$ es la que corresponde al pie cavo, en la que no existe presión del arco externo, apareciendo sólo dos 
zonas de contacto en el retropié y en la zona metatarsal. La huella de tipo D pertenece a un pie aplanado pero laxo donde el arco interno contacta sin presión con el suelo. La huella de tipo E contacta el arco interno en su totalidad, teniendo el perímetro del borde interno lineal o con una concavidad muy disminuida. La huella de tipo $\mathrm{F}$ pertenece a un pie plano acusado, donde el borde interno es convexo y en ocasiones se observa una concavidad en el lado externo debido a la pronación. La huella de tipo G, corresponde al pie plano en balancín, con una insuficiencia de apoyo del talón estrechándose la huella en la zona posterior adquiriendo forma triangular con una convexidad en el borde interno que no es reductible manualmente.

En cuanto a la patología de antepie, manteniendo la articulación subtalar en posición neutra, las superficies plantares de antepie y retropie deben ser paralelas. Si el antepie está invertido sobre el retropié se define como un antepie varo y si esta evertido en relación al retropié se define como antepie valgo ${ }^{4,23,24}$.

Debido a la importancia y frecuencia del metatarso aducto en edades infantiles ${ }^{25,26,27,28} \mathrm{se}$ observaron los siguientes signos clínicos que pueden indicar la presencia de esta patología. Se caracteriza por una aducción en conjunto del antepie a partir de la interlínea de Lisfranc, sin que exista implicación del retropie. El primer dedo en ocasiones está desviado hacia dentro y separado de los demás. El lado medial es cóncavo y el borde externo del pie es convexo con la apófisis del quinto metatarsiano prominente ${ }^{11,29}$. Puede darse un primer metatarsiano aducto que también lo clasificamos como patológico dentro de esta categoría ${ }^{25}$.

Para su valoración, se posiciona al paciente en decúbito prono con la rodilla y el tobillo flexionados a $90^{\circ}$ y la articulación subastragalina neutra. La referencia para valorar la aducción relativa del antepie a partir de la interlinea de Lisfranc es la bisección del talón que, en condiciones fisiológicas, debe pasar a través del segundo dedo o segundo espacio metatarsal. La aducción del antepie es leve si esta línea pasa por el tercer dedo o tercer espacio, moderada si pasa entre el tercer espacio y cuarto dedo y grave si pasa lateral a éste, según la clasificación de Bleck $^{26,28,30,31,32}$.
Se realizó una exploración física en camilla para evaluar la existencia de disimetrías de los miembros inferiores en aquellos alumnos en los que se observó con el pelvímetro un desnivel de las crestas iliacas en bipedestación. Se realiza con el método de Ryder colocando al alumno en decúbito supino con los pies separados $15 \mathrm{~cm}$, haciendo una ligera tracción de las extremidades comprobando si coinciden los maleolos internos. En los casos en los que se encontró alguna incongruencia, se midió con la cinta métrica la distancia entre la espina iliaca anterosuperior y la parte más prominente del maléolo tibial comparándolo con el miembro contralateral. Aunque hay algunos autores que no consideran significativas diferencias mayores a 5 milímetros ${ }^{11,33}$. Se consideró la existencia de disimetría patológica para este estudio una diferencia superior de $10 \mathrm{~mm}^{34,35,36}$.

Se valoró si presentaban el rango de amplitud articular mínima necesaria de las articulaciones del tobillo, subtalar, primer y quinto radio y primera metatarsofalángica para poder realizar una deambulación normal $1^{4,23}$. En cuanto a la articulación del tobillo es necesaria que realice una flexión dorsal mínima de $10^{\circ}$ con la articulación subtalar neutra y con la rodilla extendida. El movimiento de inversión de la articulación subtalar es dos terceras partes del movimiento total que corresponden a unos $30^{\circ}$ $35^{\circ}$ y el movimiento de eversión es una tercera parte, aunque se necesitan $4^{\circ}-6^{\circ}$ de inversión y eversión mínima de calcáneo con la supinación o pronación respectivamente de la articulación subtalar. La flexión dorsal mínima necesaria de la primera metatarsofalángica es de $65^{\circ}$. Amplitud normal de $1^{\circ}$ y $5^{\circ}$ radio: las cabezas se moverán la misma distancia por encima y por debajo del plano tranverso. Para determinar la amplitud del primer radio se coloca al paciente en decúbito prono con la articulación subtalar en posición neutra y la mediotarsiana en máxima pronación según la técnica descrita por Kevin A. Kirby ${ }^{37}$ y Root et al. ${ }^{4}$ No se valoró la movilidad de los radios centrales debido a que su movimiento es imperceptible ${ }^{4,23,37}$. En esta misma posición se observó la existencia de deformidad en flexión plantar o dorsal de algún radio metatarsal.

La variación angular de la fórmula metartarsal y los datos de la baropodometría se va- 
loraron según los criterios de Martín Rueda comenzando por el análisis de la huella plantar en estática en la que se tuvo en cuenta la evolución que va sufriendo desde la niñez hasta la edad adulta. Considerando, por tanto, pie normal aquel presenta las siguientes características. Todas las variaciones de estas características fueron consideradas patológicas:

- La prolongación del eje el estrechamiento anterior del perímetro de carga del talón se dirige en línea recta hacia el cuarto metatarsiano. Para nombrar la orientación del eje longitudinal del calcáneo se utilizaron los números de $0 \mathrm{a}$ 6 que representan los metatarsianos a los que va dirigido el vértice de carga posterior. Los números del 1 al 5 corresponden a los segmentos metatarsianos I a $\mathrm{V}$ respectivamente, anotando el valor 0 cuando el eje pasa internamente al primer radio y anotando el valor 6 cuando lo haga externamente respecto al $\mathrm{V}$ radio. Se considera normal que se dirija hacia el cuarto dedo.

- La amplitud transversa de la huella subcuboidea es la mitad o un tercio que la de talón y una cuarta parte que la del antepie. Las posibles opciones fueron: normal, aumentada o disminuida.

- El círculo de presión del talón es concéntrico sobre el centro del calcáneo. Las posibles opciones fueron: si o no.

- El círculo de presión del antepie es concéntrico sobre los metatarsianos centrales II, III y IV. Contestando: normal, hiperfunción de metatarsianos internos o hiperfunción de metatarsianos externos.

- El peso soportado por un pie es dividido igualmente entre el antepie y el retropie ${ }^{4}$ por lo que la escala colorimétrica será la misma tanto en el retropie como en el antepie. Pudiendo responder: normal, aumentada en antepie o aumentada en retropie.

- El peso en bipedestación estática es soportado equitativamente por ambos pies $^{4}$ dando lugar a una simetría de las cargas en ambos pies Respondiendo: normal, mayor carga en MID o mayor carga en MII.
Continuando con la valoración dinámica, se define el ángulo de progresión del pie como aquel formado por el eje longitudinal del pie y la línea recta de progresión de la marcha ${ }^{2,30}$ Pese a que hay ligeras diferencias en cuanto al ángulo normal de progresión del pie, los parámetros dinámicos a los 7-9 años ya son similares a los del adulto por lo que se asume, como valores normales, unas cifras entre $8^{\circ} \mathrm{y}$ $15^{\mathrm{o}} 4,11,22,30$. Por tanto se define a la marcha rotadora interna como aquella en las que el ángulo de la marcha o ángulo de Fick es inferior a $8^{\circ}$ y marcha rotadora externa a aquella en la que el ángulo de progresión del pie es mayor de $15^{\circ}$.

A los niños que presentaron un informe con un diagnóstico, a los que se les había realizado con anterioridad un estudio biomecánico concluyendo que no revelaban patología, a los que utilizaban plantillas ortopédicas y los que verbalmente manifestaron estar diagnosticados de alguna patología, fueron anotados como "diagnosticados". Al resto de los niños se les anotó como "sin diagnosticar".

Para finalizar se valoró la longitud del calzado. Para ello se tomo en bipesdestación la medida de los pies, colocándolos sobre una cartulina y dibujando la longitud, desde el talón hasta la parte distal del dedo más largo. Se recortó esta distancia y se introdujo en los zapatos hasta que contactara uno de los extremos con la punta del zapato sin que se doblara. Se consideró que el calzado era correcto si la distancia entre el otro extremo y la parte final del calzado era de al menos 10 milímetros ${ }^{13}$.

Se preguntó a los alumnos específicamente por la presencia de dolor en la espalda y miembros inferiores, dividiéndolos en 3 categorías: ausencia de dolor, dolor sólo al realizar actividad física, dolor con actividades de la vida diaria, anotando las zonas de dolor.

\section{Análisis estadístico de los datos:}

Todas las variables estudiadas son nominales excepto los grados de desviación de la línea de Helbing y los milímetros de disimetría que son cuantitativas. Para el análisis descriptivo se ha utilizado el cálculo de las frecuencias, media, mínimo, máximo y desviación típica para las variables cuantitativas, y el porcentaje para las cualitativas. El estudio de proporciones se ha realizado con el Chi-cuadrado para las va- 
riables cualitativas y se utilizó el Anova y el Bonferoni test para buscar la asociación entre la edad y los grados de desviación de la línea de Helbing. En todos los casos se consideraron diferencias estadísticamente significativas los valores de $\mathrm{p}<0,05$.

Se utilizó para la organización y análisis de los datos el programa SPSS versión 15.0 para Windows.

\section{RESULTADOS}

Se estudiaron 116 niños de entre 6 y 12 años: 53 de sexo masculino y 63 de sexo femenino, que corresponden, respectivamente, al $45,7 \%$ y al $54,3 \%$ de la muestra estudiada. La edad media fue de 8,60 años con una desviación tipica de 1,641 años. Dividiéndolos por intervalos, el $25,9 \%$ de los niños $(n=30)$ tenían 6 y 7 años, el $40,5 \%(n=47)$ tenían 8 y 9 años de edad y el $33,6 \%(n=39)$ estaban comprendidos entre los $10 \mathrm{y}$ los 12 . El peso medio fue de $31,94 \mathrm{Kg}$ con una desviación típica de $8,88 \mathrm{Kg}$. El peso mínimo fue de $17,7 \mathrm{Kg}$ y el máximo de $55 \mathrm{Kg}$. El $25,9 \%$ de los niños pesaban menos de $25 \mathrm{Kg}$, el $45,7 \%$ tenían un peso entre mayor de $25 \mathrm{Kg}$ pero menor de $35 \mathrm{Kg}$, un 17,2\% pesaban entre 35 y $45 \mathrm{Kg}$ y el peso del $12,2 \%$ era igual o mayor de $45 \mathrm{Kg}$. La talla media fue de $133,49 \mathrm{~cm}$ ( mínimo $112 \mathrm{~cm}$, máximo $155 \mathrm{~cm}$ ) con una desviación típica de $10,37 \mathrm{~cm}$. El 38,8\% de los niños median menos de $130 \mathrm{~cm}$, el 23,3\% tenian una estatura entre 130 y $137 \mathrm{~cm}$, el $18,1 \%$ entre 137 y 144 y el $19,8 \%$ median mas de $144 \mathrm{~cm}$.

La prevalencia de alteración encontrada en al menos una de las rodillas fue del $11,2 \%(n=13)$. En relación a la alteración angular de las rodillas, se observó que 5 niños $(4,3 \%)$ presentaban un genu valgo leve, 3 niños $(2,6 \%)$ presentaban genu valgo marcado y solamente se observó un caso $(0,9 \%)$ que presentara genu valgo unilateral. No se observó ningún caso de genu varo. En cuanto a la rotación de las rótulas, 3 niños $(2,6 \%)$ presentaban rótulas convergentes y 2 niños $(1,7 \%)$ divergentes.

Treinta niños $(25,9 \%)$ presentaron deformidad en algún dedo. 17 niños (14,6\%) presentaron deformidad del cuarto y/o quinto dedo. En 14 niños $(12,1 \%)$ se observó deformidad en el hallux. 9 niños $(7,8 \%)$ presentaron deformidad del segundo dedo, la misma cantidad de niños que presentaron deformidad del tercero. Las alteraciones observadas de cada dedo se detallan en la Tabla 1.

En la valoración del Foot Posture Index (FPI) se encontró como resultado que 68 niños, que corresponden al $58,6 \%$ de la población estudiada, presentaban alteración. No se observó ningún caso de postura en supinación. 55 niños $(47,4 \%)$ presentaban un FPI del pie izquierdo pronado y 11 niños $(9,5 \%)$ muy pronado. Un resultado similar se observó en el pie derecho $(48,3 \%)$ con postura en pronación y un $7,8 \%$ con un FPI muy pronado.

La prevalencia de la desviación patológica de la línea de Helbing fue del 42,2\% ( $n=49)$. En más de un $80 \%$ de los casos esta desviación se producía en eversión: $89,7 \%(n=104)$ del pie izquierdo y $82,8 \%(n=96)$ del derecho. En un $0,9 \%(n=1)$ se encontró inversión del retropié izquierdo de 2 grados, y en un $2,6 \%(n=3)$ del retropié derecho. La media de la desviación de la línea de Helbing del pie izquierdo fue de 4,68 grados en eversión con una desviación típica de 2,87 . La media de la desviación de la línea de Helbing del pie derecho fue de 3,47 grados con una desviación típica de 2,66. Los grados se exponen en las Tablas 2 y 3 . Los valores negativos corresponden a los grados de inversión.

Se observó que 65 niños (56\%) presentaban una imagen de huella plantar anormal. La frecuencia de los tipos de huella encontrados se detallan en los Gráficos 1 y 2.

La prevalencia de la patología de antepie fue del 31,9\% $(n=37)$. En el pie izquierdo se encontró una frecuencia del $26,7 \%$ de metatarso aducto que corresponde al $24,1 \%(\mathrm{n}=28)$ a un aducto leve, un hallux aducto en el 1,7\% $(n=2)$, un $0,9 \%(n=1)$ a un aducto moderado y no se ha observado ningún caso de metatarso aducto grave. Un 3,4\% $(n=4)$ de los niños presentaban antepie pronado y un $1,7 \%(n=2)$ de antepie supinado. En el pie derecho se observó una frecuencia del $22,5 \%$ de metatarso aducto que corresponde el 20,7\% $(n=24)$ al aducto leve, un $0,9 \%(\mathrm{n}=1)$ al aducto moderado que se presentó con la misma frecuencia que el hallux aducto; un $3,4 \%(n=4)$ de antepie pronado y un $1,7 \%$ $(n=2)$ de antepie supinado.

Se han encontrado 14 casos $(12,1 \%)$ de disimetría de miembros inferiores pero ninguno 


\begin{tabular}{llllll} 
Deformidad del dedo & Hallux & $\mathbf{2}^{\mathbf{0}}$ dedo & $\mathbf{3}^{\mathbf{0}}$ dedo & $\mathbf{4}^{\mathbf{0}}$ dedo & $\mathbf{5}^{\mathbf{0}}$ dedo \\
Valgo & $6,9 \%(\mathrm{n}=8)$ & $0 \%$ & $0 \%$ & $0 \%$ & $0 \%$ \\
$\begin{array}{l}\text { Varo o aducto } \\
\begin{array}{l}\text { Garra (o flexo si se } \\
\text { trata del hallux) }\end{array}\end{array}$ & $4,3 \%(\mathrm{n}=5)$ & $0,9 \%(\mathrm{n}=1)$ & $3,4 \%(\mathrm{n}=4)$ & $10,3 \%(\mathrm{n}=12)$ & $10,3 \%(\mathrm{n}=12)$ \\
\hline
\end{tabular}

Tabla 1. Porcentaje de las deformidades encontradas en los dedos.

\begin{tabular}{rlrrrr}
\hline Válidos & Frecuencia & Porcentaje & Porcentaje válido & Porcentaje acumulado \\
& $\mathbf{- 2}$ & 1 &, 9 &, 9 &, 9 \\
& $\mathbf{0}$ & 11 & 9,5 & 9,5 & 10,3 \\
$\mathbf{2}$ & 11 & 9,5 & 9,5 & 19,8 \\
$\mathbf{3}$ & 25 & 21,6 & 21,6 & 41,4 \\
$\mathbf{4}$ & 9 & 7,8 & 7,8 & 49,1 \\
$\mathbf{5}$ & 15 & 12,9 & 12,9 & 62,1 \\
$\mathbf{6}$ & 13 & 11,2 & 11,2 & 73,3 \\
$\mathbf{7}$ & 11 & 9,5 & 9,5 & 82,8 \\
$\mathbf{8}$ & 10 & 8,6 & 8,6 & 91,4 \\
$\mathbf{9}$ & 3 & 2,6 & 2,6 & 94,0 \\
$\mathbf{1 0}$ & 4 & 3,4 & 3,4 & 97,4 \\
$\mathbf{1 1}$ & 1 &, 9 &, 9 & 98,3 \\
$\mathbf{1 2}$ & 2 & 1,7 & 1,7 & 100,0 \\
\hline Total & 116 & 100,0 & 100,0 & \\
\hline
\end{tabular}

Tabla 2. Frecuencia de los grados de desviación de la línea de Helbing izquierda.

\begin{tabular}{llrrrr} 
Válidos & & Frecuencia & Porcentaje & Porcentaje válido & Porcentaje acumulado \\
& $\mathbf{- 4}$ & 1 &, 9 &, 9 &, 9 \\
& $\mathbf{- 2}$ & 2 & 1,7 & 1,7 & 2,6 \\
& $\mathbf{0}$ & 19 & 16,4 & 16,4 & 19,0 \\
$\mathbf{2}$ & 24 & 20,7 & 20,7 & 39,7 \\
$\mathbf{3}$ & 16 & 13,8 & 13,8 & 53,4 \\
\hline $\mathbf{4}$ & 13 & 11,2 & 11,2 & 64,7 \\
$\mathbf{5}$ & 12 & 10,3 & 10,3 & 75,0 \\
$\mathbf{6}$ & 13 & 11,2 & 11,2 & 86,2 \\
$\mathbf{7}$ & 8 & 6,9 & 6,9 & 93,1 \\
$\mathbf{8}$ & 4 & 3,4 & 3,4 & 96,6 \\
$\mathbf{9}$ & 4 & 3,4 & 3,4 & 100,0 \\
\hline
\end{tabular}

Tabla 3. Frecuencias de los grados de desviación de la línea de Helbing derecha. 


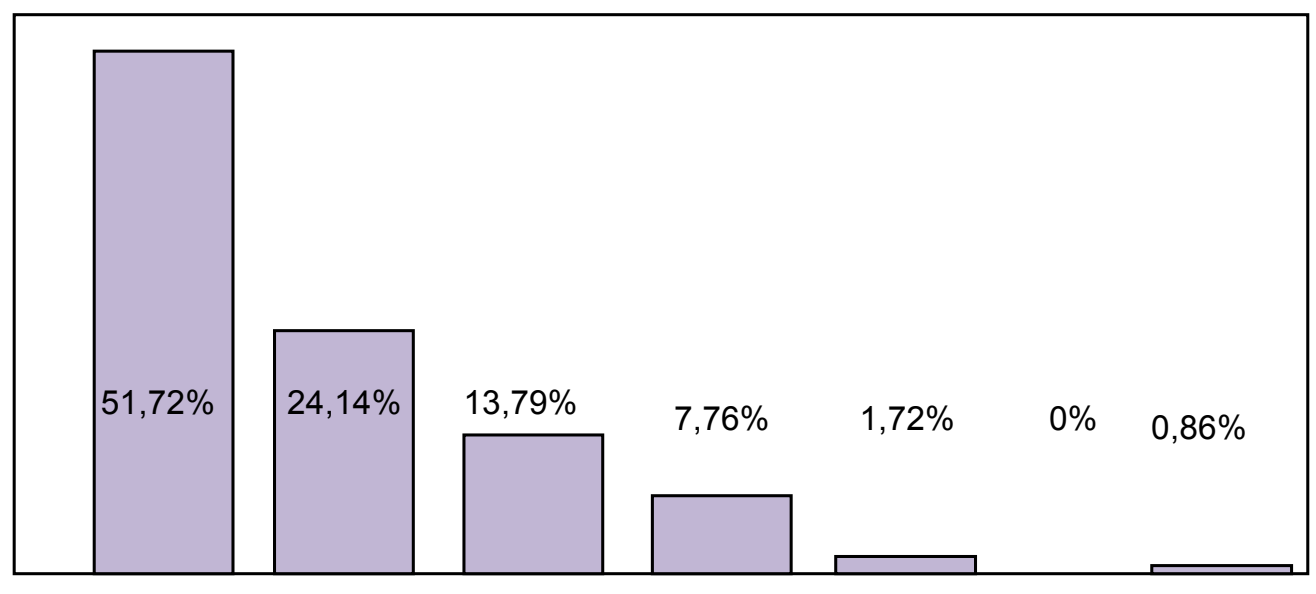

A

B

C

D

$\mathrm{E}$

$\mathrm{F}$

G

Gráfico 1. Diagrama de barras del porcentaje del tipo de huella izquierda.

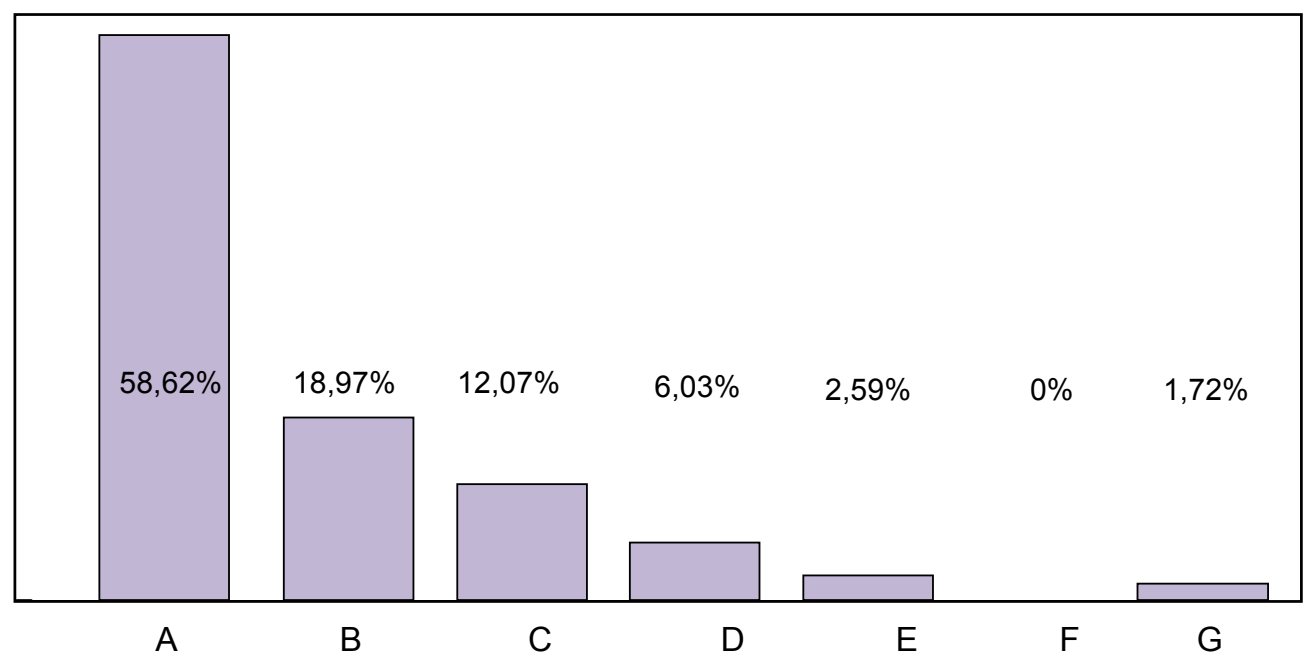

Gráfico 2. Diagrama de barras del porcentaje del tipo de huella derecha.

\begin{tabular}{llllll} 
Válidos & $\mathbf{0} \mathbf{~ m m}$ & Frecuencia & Porcentaje & Porcentaje válido & Porcentaje acumulado \\
\cline { 2 - 4 } & $\mathbf{3} \mathbf{~ m m}$ & 1 & 87,9 & 87,9 & 87,9 \\
& $\mathbf{4} \mathbf{~ m m}$ & 2 &, 9 &, 9 & 88,8 \\
& $\mathbf{5} \mathbf{~ m m}$ & 7 & 1,7 & 1,7 & 90,5 \\
& $\mathbf{8} \mathbf{~ m m}$ & 1 & 6,0 & 6,0 & 96,6 \\
& $\mathbf{1 0} \mathbf{~ m m}$ & 3 &, 9 &, 9 & 97,4 \\
\hline Total & 116 & 2,6 & 2,6 & 100,0 \\
\hline
\end{tabular}

Tabla 4. Porcentajes observados de los milímetros de disimetría. 
fue patológico. La media fue de 0,72 $\mathrm{mm}$ con una desviación típica de $2,12 \mathrm{~mm}$. La frecuencia de una mayor longitud del miembro inferior izquierdo fue de un 10,3\% $(\mathrm{n}=12)$ de los casos. La frecuencia de mayor longitud del miembro inferior derecho fue del 1,7\% $(n=2)$ (Tabla 4).

En cuanto a las alteraciones de la movilidad, según la lateralidad un $12,9 \%(n=15)$ de los niños presentaron limitación de la movilidad en alguna articulación del pie izquierdo. La articulación más frecuentemente afectada fue la del tobillo, en un 5,2\% $(n=6)$. La primera art. metatarsofalángica, con resutados similares, se observó en el 4,3\% $(n=5)$. El 2,6\% $(n=3)$ tenía limitación de la art. subtalar y por último, solo se vio un caso de limitación del primer radio.

Un $10,3 \%(n=12)$ de los niños evaluados presentaron limitación de la movilidad de alguna articulación del pie derecho. La articulación con movilidad limitada con más frecuencia fue la primera metatarsofalángica en un $4,3 \%(n=5)$ de los niños. El 3,4\% $(n=4)$ tenían limitada la flexión dorsal del tobillo. La art. subtalar se encontraba limitada en el 2,6\% $(n=5)$. No se encontraron alteraciones en el resto de las articulaciones del pie.

En relación a la presencia de radios en flexión plantar, la prevalencia observada ha sido de $15,5 \%(n=18)$ pero solo se han encontrado casos en los que estaban afectados el primer radio, con una frecuencia del $13,8 \%(n=16)$ y el primer y quinto a la vez con una frecuencia del $1,7 \%(n=2)$. No se observaron alteraciones de los radios centrales ni del quinto exclusivamente.

En relación a los resultados obtenidos en el análisis de la baropodometría estática, se obtuvieron los siguientes resultados:

Un 66,4 \% $(n=77)$ de los niños presentaron desviación de la prolongación del eje del talón en uno de los dos pies. Según la lateralidad, en el pie izquierdo la frecuencia de la alteración del eje es de un 19\% hacia el segundo metatarsiano, un $17,2 \%$ hacia el tercero, un $10,3 \%$ hacia el primero, se observó el mismo porcentaje $(2,6 \%)$ en la prolongación que se dirigía medialmente al primer metatarsiano y hacia el quinto; por último, en un $1,7 \%$ se desviaba lateralmente al quinto metatarsiano. En el pie derecho los porcentajes de alteración son de un $24,1 \%$ hacia el tercer metatarsiano, un $11,2 \%$ hacia el primero, 6,9\% hacia el segundo, 2,6\% hacia la zona medial del primer metatarsiano y un 1,7\% hacia la zona lateral del quinto.

La presión de la huella subcuboidea se observó alterada, aumentada o disminuida, en el $80,2 \%(n=93)$ de los casos, en al menos un pie. La alteración más frecuente fue la disminución de la anchura de la imagen, en un 51,7\% $(n=60)$ en el pie izquierdo de los niños estudiados y en un $54,3 \%(n=63 \%)$ en el pie derecho. Una anchura aumentada fue observada en un $13,8 \%$ $(n=16)$ en el pie izquierdo y un $11,2 \%(n=13)$ en el pie derecho.

La prevalencia de la alteración del reparto de presiones fue del 94,8\% $(n=110)$ de los niños en uno o en los dos pies. Respecto al pie izquierdo se observó que un 33,6\% $(n=39)$ presentaban alteración de la presión que ejercía el talón, en el $36,2 \%(n=42)$ de los niños se ponía de manifestó una hiperfunción de los metatarsianos internos y un 5,2\% $(n=6)$ una hiperfunción de los externos. El porcentaje de niños que tenían alterada la presión del talón derecho fue del $38,8 \%(n=45)$. En cuanto al antepie, un $41,4 \%$ $(n=48)$ tenían una hiperfunción de los metatarsianos internos y un 6,9\% $(n=8)$ hiperfunción de los externos.

En el miembro inferior izquierdo, la alteración más frecuente del reparto del peso de cada pie fue el aumento de presión en el antepie que se observó en un 38,8\% $(n=45)$ de los niños evaluados y un aumento del peso del retropié en un $25,9 \%(n=30)$. En el miembro inferior derecho, el 40,5\% $(n=47)$ de los niños presentaron aumento de presión en el antepie y un 18,1\% $(n=21)$ aumento de presión en el retropié.

Se observó que el 48,3\% de los niños presentaba una asimetría de las cargas en ambos pies, siendo mayor la presión sufrida por el pie izquierdo (40,5\%).

El ángulo de progresión de la marcha estaba alterado en el $15,6 \%(n=18)$ de los niños correspondiendo en su mayoría a una marcha aducta $(14,7 \%)$ y al $0,95 \%$ de los casos a una marcha en abducción.

La prevalencia de alteración de alguna de las fases de la dinámica en cualquiera de los dos pies fue del 75\% ( $n=87)$. Dividiéndolo por fases, se encontró patología durante el contacto de talón en el 36,2\% $(n=42)$ de los niños, durante el apoyo total en el $34,5 \%(n=40)$, durante la 
fase de propulsión en el $56 \%(n=65)$ y durante el despegue en el 33,6\% ( $n=39)$. El contacto de talón del pie izquierdo fue anormal en el 30,1\% de los niños evaluados, un 26,7\% (n=31) realizan el contacto de talón izquierdo en pronación y el 3,4\% $(n=4)$ en excesiva supinación. El contacto de talón del pie derecho fue anormal en el $23,3 \%$, el 21,6\% $(n=25)$ lo realiza en pronación y el $1,7 \%(n=2)$ en supinación excesiva. La fase de apoyo total del pie izquierdo estaba alterada en el $20,7 \%$ de los niños. El 15,5\% $(n=18)$ en excesiva pronación y el 5,2\% $(n=6)$ en excesiva supinación. La fase de apoyo total del pie derecho se encontró alterada en el $28,5 \%$ de los niños, el 23,3 $(n=27)$ en excesiva pronación y el 5,2\% $(n=6)$ en excesiva supinación.

La fase de propulsión del pie izquierdo fue patológica en el $38,8 \%$, un $22,4 \%(n=26)$ de los niños realizan la propulsión del pie izquierdo en excesiva supinación y el $16,4 \%(n=19)$ en excesiva pronación. La fase de propulsión del pie derecho fue patológica en el $45,7 \%$ de los niños, un $25,9 \%(n=30)$ la en excesiva pronación y un $19,8 \%(n=23)$ en supinación. La prevalencia de despegue anormal del pie izquierdo, es decir, con los metatarsianos centrales fue del $29,3 \%(n=34)$, mayor que en el pie derecho que fue del $19 \%(n=22)$.

De los 116 niños estudiados, $8(6,9 \%)$ estaban diagnosticados o se les había realizado previamente un estudio similar.

Un 75\% de los niños ( $\mathrm{n}=87$ ) llevaban un calzado con una longitud inferior a la necesaria.

El 39,7\% $(n=46)$ de los niños referían dolor en alguna zona de la espalda o las extremidades inferiores. Un 10,3\% presentaban dolor de es- palda. No hubo ningún caso que presentara dolor de cadera. El dolor de rodilla se dio en un $6 \%$ y $7,8 \%$ en la pierna izquierda y derecha respectivamente. El $6 \%$ de los niños presentaban dolor en la pierna izquierda y el $7,8 \%$ en la derecha. El porcentaje más elevado de dolor se encontró en el pie, el 19,8\% en el izquierdo y el $22,4 \%$ en el derecho. La frecuencia del dolor según la zona y la relación con la actividad física se muestran en la Tabla 5.

En función de la zona del dolor en el pie, se observó que el $6 \%(n=7)$ de los niños refería dolor en el tobillo de, por lo menos, alguno de los pies. El 4,3\% $(n=5)$ en el talón, en este caso, siempre bilateral. El 2,6\% $(n=3)$ referían dolor de al menos uno de los pies en la zona del arco lateral interno y el 1,7\% $(\mathrm{n}=2)$ en la zona metatarsal.

En relación con el sexo, solamente se han encontrado diferencias estadísticamente significativas en la presencia de alteración en la fase de propulsión de la marcha $(p=0,02)$. No se han encontrado diferencias en la relación del peso con la presencia de patología.

Las diferencias estadísticamente significativas encontradas en relación con la edad solo se observaron en la prevalencia de retropié derecho patológico $(\mathrm{p}=0,02)$ y en la alteración del eje de progresión del talón en alguno de los dos pies $(p=0,046)$. En concreto, respecto a la alteración del eje de progresión del pie izquierdo se obtuvo un valor de $\mathrm{p}$ de 0,014 .

Se han encontrado diferencias estadísticamente significativas en la prevalencia de radios desviados en flexión plantar $(\mathrm{p}=0,01)$ y la deformidad del cuarto y quinto dedo $(p=0,034)$ con relación a la variación angular de la fórmu-

\begin{tabular}{lll} 
Zona de dolor & $\begin{array}{l}\text { Dolor con actividades } \\
\text { de la vida diaria }\end{array}$ & $\begin{array}{l}\text { Dolor con actividad } \\
\text { física moderada }\end{array}$ \\
\hline Espalda & $8,6 \%(\mathrm{~N}=10)$ & $1,7 \%(\mathrm{~N}=2)$ \\
\hline Rodilla IZDA & $6 \%(\mathrm{~N}=7)$ & $0 \%$ \\
\hline Rodilla DCHA & $7,8 \%(\mathrm{~N}=9)$ & $0 \%$ \\
\hline Pierna IZDA & $4,3 \%(\mathrm{~N}=5)$ & $1,7 \%(\mathrm{~N}=2)$ \\
\hline Pierna DCHA & $6 \%(\mathrm{~N}=7)$ & $1,7 \%(\mathrm{~N}=2)$ \\
\hline Pie IZDO & $16,4 \%(\mathrm{~N}=19)$ & $3,4 \%(\mathrm{~N}=4)$ \\
\hline Pie DCHO & $19 \%(\mathrm{~N}=22)$ & $3,4 \%(\mathrm{~N}=4)$ \\
\hline
\end{tabular}

Tabla 5. Frecuencia del dolor según las zonas y la relación con la actividad física. 
la metatarsal. No se han encontrado diferencias significativas en relación al resto de patologías de antepie ni con alteraciones en la dinámica.

Se han encontrado diferencias estadísticamente significativas en relación a la articulación que no presentaba la amplitud mínima de movimiento del pie izquierdo con la fase de apoyo total del pie homolateral, en la que la significación asintótica bilateral es de 0,03 . No se han encontrado diferencias estadísticamente significativas en la relación de la presencia de articulaciones sin amplitud mínima de movimiento del pie izquierdo con otras alteraciones de la dinámica.

Solamente se han encontrado diferencias estadísticamente significativas en relación a la articulación del pie derecho que no presentaba la amplitud mínima de movimiento con la fase de contacto de talón del pie izquierdo $(p=0,04)$, con el resto de alteraciones de la dinámica no se han encontrado diferencias.

Se ha relacionado la edad con la desviación de los grados de la línea de Helbing del pie derecho dando como resultado diferencias estadísticamente significativas $(\mathrm{p}=0,01)$. Existe entre ellas una relación lineal $(p=0,039)$. En relación al pie izquierdo no se han encontrado diferencias.

\section{DISCUSIÓN}

Tras el estudio realizado hemos observado que al 93\% de los escolares nunca les habían realizado una exploración podológica y un estudio biomecánico similar previo. Otros autores han encontrado datos similares, igual de sorprendentes, en los que la cifra de alumnos diagnosticados y con tratamiento oscilaba entre el 4 y el $14 \%{ }^{11,38,39,40}$.

Algunos autores estiman que algo más del 70 $\%$ de los escolares y adolescentes presentan alguna patología musculoesquelética ${ }^{41,42}$. Hemos recogido datos similares, aunque los mayores porcentajes de alteración se dieron en la baropodometría estática, en el que casi un $95 \%$ de los niños presentaban una alteración del reparto de presiones de la huella plantar. Ocho de cada diez alumnos evaluados tenían alterada la presión de la huella subcuboidea, lo más frecuente fue su estrechamiento e incluso ausencia. La prolongación del eje del talón, alterado en más de la mitad de los niños, tuvo tendencia a desviarse hacia los radios mediales; con el mismo porcentaje hemos observado una asimetría de las cargas que soportan ambos miembros inferiores, siendo normalmente el pie izquierdo el que más peso soporta.

Sin embargo, a pesar de la alta prevalencia de patología estática, la alteración de alguna de las fases de la dinámica, aunque también muy elevada, ha sido observada en un porcentaje algo inferior. Lo más frecuente, afectando al 50\% de los niños fue la alteración de la propulsión. Las otras tres fases de la dinámica estuvieron afectadas por igual entorno al $34 \%$ cada una. Con mayor frecuencia en pronación. No se han podido comparar estos resultados con otras publicaciones debido a que los autores se han centrado en la valoración del pico de presión, el tiempo de contacto o la velocidad del paso, datos no valorados en este estudio. La mayoría de esos trabajos se han realizado además, a través de las plantillas electrónicas pudiendo diferir los datos con los encontrados en la plataforma de presiones, a causa de que estas últimas no tienen en cuenta las variaciones de los pies con las ortesis o el calzado ${ }^{43,44,45}$.

La frecuencia de huella anormal (56\%) coincide con la media de los valores encontrado por otros autores, que estimaron prevalencias entre el 35 y el $92 \% 111,42,46,47,48,49$. Aproximadamente el $35 \%$ de los niños presentaban huella cava B o C según la clasificación de Martín Rueda ${ }^{5}$, resultados que duplican e incluso triplican a los de otras publicaciones ${ }^{11,17,47}$. Algunos autores buscaron la prevalencia del pie cavo morfológico dando lugar a resultados aún menores que llegan incluso al 3,6\% $0^{49,50,51}$. Estas diferencias pueden deberse a los diversos criterios empleados ya que en este trabajo, solo hemos valorado para esta variable, la huella y no la morfología del pie.

Muchos autores estiman la prevalencia del pie plano entre el 20 y el $30 \%$ de los niños $17,38,48,52$ y los resultados de este estudio han sido aproximadamente de la mitad, similares a Zurita ${ }^{47}$, Ferrer et al. ${ }^{49}$ y Baar et $a^{50}$. En relación a los diferentes grados de huella plana, casi todos los casos (aproximadamente un 7\% de los niños) presentaban el tipo D que equivaldría al primer grado, coincidiendo con Zarate et al. ${ }^{38}$ 
y Revenga et $a l .{ }^{51}$ aunque sus resultados fueron mucho mayores, llegando al $63 \%$ en este tipo de huella. En el resto de los casos, la prevalencia va disminuyendo según aumenta el grado de alteración. Hubo pocos o ningún caso de los tipos $\mathrm{E}, \mathrm{F}$ y G $\left(2^{\circ}, 3^{\circ}\right.$ y $4^{\circ}$ grado respectivamente $)$. Hemos encontrado una altísima variabilidad en los resultados publicados por otros autores, desde el 2.7 hasta el $80 \%$, que puede ser debido a la falta de uniformidad en los criterios utilizados y a la gran diversidad de los grupos estudiados. Posiblemente estas diferencias son también debidas a la modernización de los instrumentos empleados para el análisis en este estudio desde la publicación de esos trabajos, que permiten valorar de una manera más detallada y exahustiva. Así es que, en la gran mayoría de ocasiones, los autores utilizaron el podoscopio o las podografías. Las diferencias también pueden deberse a que los niños evaluados practicaban deporte o eran algo mayores que los de este estudio $^{11,17,38,40,48,49,51,52,53,54,55}$. Casi todos estos trabajos publicados confirman la mayor prevalencia de pie plano en niños que en niñas, pero en este trabajo no se han encontrado diferencias significativas en relación con el sexo.

También es llamativo el alto número de niños que estaban utilizando un calzado corto, algo superior a otros estudios que encontraron alrededor de un $60 \%$ de prevalencia ${ }^{56,57}$. A pesar de que en todos los casos la morfología del calzado era adecuada, solo el 25\% tenía una longitud correcta. No hemos encontrado ningún caso que llevara calzado excesivamente grande, al contrario que Ramos ${ }^{11}$ y el trabajo publicado por la American Academy of Orthopaedic Surgeons ${ }^{57}$ que sí encontraron un pequeño porcentaje.

Un $42 \%$ de los escolares presentaban una desviación de la línea de Helbing predominantemente en eversión (entre el 80 y 90\%). La prevalencia de patología es inferior en un trabajo publicado por Revenga et al. $(33,3 \%)^{51}$ pero puede deberse a que el autor considera patológica una desviación de $7^{\circ}$ en valgo; sin embargo, otros autores encontraron frecuencias similares, entre el 25 y el $46 \%{ }^{11,49,53}$. La desviación en inversión ha sido infrecuente, y hemos obtenido una prevalencia de aproximadamente la mitad que otros trabajos debido probablemente, a que la edad media de los niños evaluados por ellos era algo superior, de unos 12 años y practicaban deporte $^{17,49}$. Molano en 2004 encontró una prevalencia de retropié varo del $18 \%{ }^{53}$. Así mismo hemos encontrado diferencias estadísticamente significativas del pie derecho en relación con la edad, pero no se han encontrado en el pie izquierdo.

En relación con la desviación del retropié, encontramos que más de la mitad de los niños presentaban el índice de postura del pie alterado, siempre en pronación aunque un ligero porcentaje, entorno al 8-9\% eran pies muy pronados; resultados ligeramente superiores a un trabajo similar hecho por Ramos en 2007: en el que observó un 36\% de prevalencia en pronación ${ }^{11}$.

En 1998 Arocena et al ${ }^{58}$ publicaron que el 6.2 $\%$ de los motivos de consulta en pediatría de Atención Primaria era el dolor musculoesquelético. Hay una discreta diversidad, según los distintos autores, en relación a la presencia de dolor o sintomatología y según la edad, que va desde el 5 al 37\% $0^{41,47,50,59,60}$. Muchos estudios están orientados a buscar la sintomatología de los pies planos ${ }^{38,50,54,61}$. Los resultados de este estudio, independientemente de si coexisten con patología o no, son ligeramente superiores a la media de estos trabajos, ya que se ha obtenido que aproximadamente cuatro de cada diez niños presentaban dolor. Las localizaciones más frecuente fueron el pie (20\%), seguido de la espalda con la mitad del porcentaje coincidiendo con García $^{59}$, por último las piernas, con un $7 \%$ resultado muy distante al del trabajo publicado por Evans et al. en 2004 en el que encuentra un $37 \%{ }^{60}$. No hubo ningún caso con dolor de cadera. El miembro inferior derecho fue ligeramente el más afectado pero sin diferencias significativas.

Del total de la patología de antepie hallada $(32 \%)$ la más frecuente fue el metatarso aducto leve, entorno al $25 \%$. Ebri ${ }^{28}$ observó en su estudio una prevalencia del 1 al $10 \%{ }^{28}$ Esto entra en discordancia con otros trabajos publicados que afirman que 1a prevalencia del metatarso aducto es 1 de cada mil nacimientos vivos y que se corrige espontáneamente durante los primeros años de $\operatorname{vida}^{25,61}$. La pronación y la supinación del antepie se vieron en pocas ocasiones.

Según Rueda ${ }^{5}$, las variaciones angulares de la fórmula metatarsal provocan cambios de torsión de la extremidad inferior y condicionan 
la posición del pie durante la marcha. Afirma que las fórmulas metatarsales oblicuas se relacionan con marcha en rotación interna, y las fórmulas transversas se relacionan con marcha en rotación externa. En este estudio, el 11,2\% de los niños presentaba una fórmula metatarsal transversa, el 5,2\% en rueda dentada y el 4,3\% oblicua, pero en ninguno de ellos se han encontrado diferencias estadísticamente significativas en relación con patología en la dinámica. Solo hemos encontrado diferencias en relación a la deformidad del cuarto y quinto dedo que fueron además las más frecuentes del $25 \%$ de los niños que presentaron deformidades de los dedos. El hallux se vio alterado en un porcentaje similar y algo inferior a Benedetti et al. ${ }^{62}$. Hemos encontrado datos mucho mayores que Ramos $^{11}$ que encontró una prevalencia de alteración digital del 2,5\%, o la American Academy of Orthopaedic Surgeons ${ }^{57}$ que encontró un $3,3 \%$ de hallux valgus.

Aproximadamente en 1 de cada 10 niños se observó una limitación de la movilidad de alguna articulación, muy distante a los encontrados por Benedetti et $a l .{ }^{62}$ en niños ligeramente mayores, que lo observó en un $68 \%$ de prevalencia de limitación funcional. Es curioso que se encontraran diferencias significativas en la limitación de alguna articulación del pie derecho con la fase de contacto de talón del izquierdo, y en la limitación de alguna articulación del pie izquierdo con la fase de apoyo total del pie derecho.

Los únicos radios afectados fueron el primero y el quinto que se encontraron en un $15 \%$ en flexión plantar. No se ha encontrado bibliografía al respecto en niños de esta edad por lo que no se han podido comparar los resultados.

El porcentaje de niños que presentaban el ángulo de progresión de la marcha dentro de los valores normales fue del $84 \%$ ligeramente inferior a los encontrados por Ramos ${ }^{11}$ que llega al 94\% pero superior a otros trabajos publicados en los que este porcentaje descendía al $58 \%$ e incluso al $35^{41,42}$. Hemos obtenido menos prevalencia de alteración del ángulo de progresión de la marcha que Ibañez et al. ${ }^{41}$, casi el $15 \%$ una marcha en aducción o convergente frente a su $23 \%$, y un escaso número de niños con marcha en abducción comparado con el número de casos encontrados por él en el que se duplican.
Esta diferencia puede deberse, tal y como afirman estos autores, a que existe una relación entre la edad y la marcha convergente, siendo ésta más frecuente en niños más pequeños.

La frecuencia encontrada de disimetría, en ningún caso patológica, fue ligeramente superior a otros trabajos ${ }^{48}$, debido posiblemente a que los niños evaluados en este estudio eran de menor edad, y concuerda con Gonzalez ${ }^{36}$ al afirmar que diferencias tan mínimas pueden deberse a errores en la medición o al proceso dinámico que se produce durante la maduración esquelética. El miembro más largo fue casi siempre el izquierdo y nunca se superó la diferencia de más de un centímetro universalmente más aceptado para necesitar tratamiento, puesto que afirman que diferencias de más de 1,5 ó $2 \mathrm{~cm}$ no provocan trastornos funcionales de la marcha ni repercusión sobre la columna ${ }^{33,34,35,36}$.

Los resultados en relación a la patología de rodilla coinciden con estudios similares en el que un alto porcentaje de los escolares no presentan patología angular ni rotaciona $1^{17,49}$. La alteración más frecuente coincidiendo con toda la bibliografía consultada ha sido el genu valgo. Unos autores han encontrado una prevalencia menor en niños más pequeños ${ }^{16,51,53}$ y el porcentaje publicado por otros, en niños de la misma edad o ligeramente superior (de 10 a 14 años), duplica los resultados de este estudio por lo que podemos pensar contrariamente a lo esperado que esta patología no disminuye con la edad, aunque no hemos encontrado diferencias estadísticamente significativas al respecto ${ }^{17,48,49}$. La prevalencia más alta es la publicada en un trabajo realizado por Ramos $(32 \%)^{11}$ En cuanto al genu varo no se detectó ningún caso, al igual que otro estudio realizado con niños de preescolar ${ }^{16}$ pero, sí difieren con la prevalencia encontrada en niños deportistas de mayor edad, que va desde un $4 \%$ en niños de 7 a 14 años y aumenta hasta el $14 \%$ en adolescentes entre 10 y 20 años, por lo que podemos pensar que a medida que aumenta la edad y la actividad deportiva se puede elevar el riesgo de padecer genu varo $^{11,17,48,49,53}$. Estas diferencias pueden ser debidas también a los diferentes criterios utilizados para considerarlo patológico. Los casos de convergencia o divergencia rotuliana han sido muy escasos, aproximadamente del $2 \%$, incluso algo inferiores a los encontrados por Ferrer ${ }^{49}$ 
Sin embargo, en niños algo menores, Chauca en 2008 halló un $67 \%$ de niños con deformidad torsional ${ }^{42}$.

El número tan elevado de patologías encontradas puede deberse a que los criterios de normalidad descritos por Root et al. que hemos utilizado son muy rigurosos y restrictivos, como afirma Kevin A. Kirby ${ }^{63}$ Es posible también, que no haber utilizado otros métodos como plantillas instrumentalizadas o la radiología para realizar los diagnósticos, puedan aumentar el número de alteraciones observadas. Tanto los signos clínicos como todos los instrumentos empleados están validados y son fiables para realizar un primer cribado y así conocer el estado de salud de los pies de los escolares. Los resultados se han comunicado a los padres de los alumnos a través de informes, recomendando en los casos necesarios, un estudio biomecánico más exahustivo para confirmar o descartar las alteraciones encontradas. Se han propuesto además charlas informativas en el colegio sobre el desarrollo del pie, prevención de patologías y recomendaciones del calzado. Este trabajo nos ha permitido abrir futuras líneas de investigación, extendiéndolo a otros colegios para cono- cer el estado de salud de los pies de los niños del concejo, hacer un seguimiento o ver la evolución de estas patologías y su repercusión en la edad adulta.

\section{CONCLUSIONES}

Los resultados observados confirman la gran prevalencia de patologías en el pie infantil no diagnosticadas, debido al altísimo porcentaje de niños a los que nunca se les había realizado una exploración podológica. Fue llamativo el número de niños con alteraciones en la dinámica y en la huella, en la mayoría de ocasiones, por una excesiva pronación. Sigue existiendo una gran variabilidad en los resultados publicados debido a la falta de uniformidad en los criterios o en las diferentes técnicas utilizadas. Menos frecuentes fueron las alteraciones angulares o rotacionales de las rodillas. Muchas alteraciones musculoesqueléticas en edades infantiles no suelen producir sintomatología ni dolor, de ahí la importancia de realizar revisiones podológicas y estudios biomecánicos periódicos para evitar complicaciones en la edad adulta.

\section{BIBLIOGRAFÍA}

1. Losada P. Alteraciones de los miembros inferiores: deformidades angúlares, torsionales, alteraciones de la marcha y dismetrías. Pediatr Integral 2002; 6(5): 397-412.

2. Calzadilla V, Castillo I, Blanco J, González E. Desviaciones torsionales de los miembros inferiores en niños y adolescentes. Rev Cubana Med Gen Integr 2002; 18(5).

3. Serrano S. Desarrollo angular y rotacional de los miembros inferiores en escolares entre 3 y 10 años. Estudio de dos poblaciones diferentes [ tesis doctoral]. Bogotá: Universidad Nacional de Colombia; 2011.

4. Root M, Orien W, Weed J. Función normal y anormal del pie. Barcelona: Base; 2012.

5. Rueda M. Los desequilibrios del pie. Barcelona: Paidotribo; 2011.

6. Rao UB, Joseph B. The influence of footwear on the prevalence of flatfoot. J Bone Joint Surg. 1992; 74-B(4): 525-527.

7. Kurup HV, Clark CIM, Dega RK. Footwear and orthopaedics. J Foot Ankle Surg. 2012; 18(2): 79-83.

8. Gentil I. Podología preventiva: niños descalzos igual a niños más inteligentes. Rev Int Cienc Pod. 2007; 1(1): 27-34.

9. Staheli L. Plano valgus foot deformity. Current status. J Am Podiatr Med Assoc. 1999; 89(2): 94-99.

10. American Academy of Orthopaedic Surgeons [Internet]. Illinois: American Academy of Orthopaedic Surgeons; 2009 [acceso el 3 de febrero de 2013]. News and Journals [3 páginas]. Disponible en: http:// www.aaos.org/news/aaosnow/mar09/clinical5.

11. Ramos J. Detección precoz y diagnóstica de alteraciones podológicas en población escolar [tesis doctoral]. Sevilla: Universidad de Sevilla; 2007.

12. Moreno JL. Podologia general y biomecánica. Barcelona: Masson; 2003.

13. Instituto Biomecánico de Valencia. El pie calzado. Guía para el asesoramiento en la selección del calzado infantil. Valencia: Generalitat Valenciana; 1999.

14. Jiménez R, Martín ML, Pérez SM. Estudio técnico del calzado. El Peu 2003; 23(3): 140-46. 
15. Santonja F. Reconocimiento del aparato locomotor durante la edad escolar. En: Universidad de Murcia, Secretariado de Publicaciones e Intercambio Científico. Valoración médico-deportiva del escolar. $1^{\text {a }}$ ed. Murcia: Universidad de murcia; 1992. 259-277.

16. Griman AC. Frecuencia de defectos ortopédicos en preescolares. Consulta de niños sanos. Ambulatorio "Daniel Camejo Acosta" [trabajo fin de grado] Barquisimeto: Universidad centro occidental "Lisandro Alvarado"; 2001.

17. Nicasio JJ, Díaz FJ, Sotelo F, Melchor MT. Prevalencia de alteraciones músculo-esqueléticas en jóvenes preparatorianos. Acta Ortopédica Mexicana 2003; 17(2): 68-73.

18. Barbero AM, Ruisánchez S, Vaquerizo MJ, Villa M. Problemas ortopédicos prevalentes en el niño. En: Asociación Española de Pediatría. Manual del residente en pediatría y sus áreas específicas. Vol. 2. Madrid: Norma; 1997. 1169-1182.

19. Romero D, León MR. Ortesis en las alteraciones ortopédicas de miembro inferior. En: XVI Jornadas científicas de la Sociedad Española de Rehabilitación Infantil. Sevilla: Sociedad Española de Rehabilitación infantil; 2011. 34-37.

20. Redmon A. Foot Posture Index. User guide and manual [parte de una página web] Agosto 2005 disponible en: www.leeds.ac.uk/medicine/fFASTER/FPI

21. Padilla V. Evaluación del tratamiento ortopodológico en el pie plano flexible en niños de 3 a 5 años [trabajo fin de máster]. Madrid: Reduca: Series trabajos fin de máster. 2011; 3(2): 265-288.

22. Viladot A. Quince lecciones sobre patología del pie. Barcelona: Springer-Verlag Ibérica; 2000.

23. Consejo general de colegios de podólogos. Guía práctica de protocolos de exploración y biomecánica. Madrid: Consejo General de Colegios Oficiales de Podólogos; 2010.

24. Glasoe WM, Allen MK, Ludewig PM. Measuring forefoot alignment with a table-mounted goniometric device. Australian Journal of Physiotherapy 2002; 48: 51-53.

25. Dalal A, Pimentel-Tejeda A, Kim A. Literature Review of Metatarsus Adductus in Children. NYCPM. Student Association Podiatric Medical Review 2011-2012; 20:24-29.

26. Connors JF, Wernick E, Lowy LJ, Falcone J, Volpe RG. Guidelines for evaluation and management of five common podopediatric conditions. J Am Podiatr Med Assoc. 1998; 88(5):206-22.

27. Brink D. Childhood foot and leg problems. Pediatr Ann. 1976; 5(4): 60-80.

28. Ebri JR. El pie infantil: crecimiento y desarrollo. Deformidades más frecuentes: pie doloroso. Pediatr Integral. 2002; 6(5): 431-452.

29. Rotés MI, Gonzalez L. El pie en crecimiento Rev Esp Reumatol 2003; 30(9):516-35.

30. Castillo JM: Efecto de los soportes plantares con cuña rotadora externa en las marchas rotadoras internas en el niño [tesis doctoral] Sevilla: Universidad de Sevilla, 2007.

31. Martínez-Sahuquillo ME. Medidas de resultados en las alteraciones ortopédicas infantiles de los miembros. En: XVI Jornadas científicas de la Sociedad Española de Rehabilitación Infantil. Sevilla: Sociedad Española de Rehabilitación infantil; 2011. 18-20.

32. Bleck EE. Metatarsus adductus. Classification and relationship to outcomes of treatment. J Pediatr Orthop. 1983; 3(1): 2-9.

33. Jurado A, Medina I. Manual de pruebas diagnósticas. Traumatología y ortopedia. $1^{\circ}$ ed. Barcelona: Paidotribo; 2002.

34. Starkey C, Brown SD, Ryan JL. Evaluación de la postura. En: Starkey C. Patología ortopédica y lesiones deportivas. Guía de examen. $2^{\circ}$ edición. Buenos Aires: Panamericana; 2012. 77-103.

35. López J. Otros protocolos. Patología de la espalda y miembro inferior. Bol Pediatr. 2006; 46: 327-335.

36. González P. Tratamiento actual de las dismetrías y de las alteraciones rotacionales del miembro inferior. En: XVI Jornadas científicas de la Sociedad Española de Rehabilitación Infantil. Sevilla: Sociedad Española de Rehabilitación infantil; 2011.38-42.

37. Kirby K. Test y técnicas clínicas. En: Precision Intricast. Biomecánica del pie y de la extremidad inferior II: Artículos de Precision Intricast, 1997-2002. Arizona: Precision intricast; 2002. 150-160.

38. Zárate A, Pereira MA, Ibarrola J, Kikuchi A, Sanabria L. Prevalencia de pie plano en niños escolares de Asunción y Gran Asunción. An Fac Cienc Med. 2009; 42(2): 13-18.

39. Pfeiffer M, Kolz R, Ledl T, Hauser G, Sluga M. Prevalence of flat foot in prescholar-aged children. Pedriatrics. 2006; 118: 634-640.

40. García A, Martín F, Carnero M, Gomez E, Gomez J, Fernández J. Flexible flat feet in children: a real problem? Pediatrics 1999; 103(6): 84.

41. Ibañez A, Baar A, Gana N. Cambios fisiológicos de la rotación de la marcha durante el desarrollo. Rev Chil Pediatr. 2008; 79(1): 45-49. 
42. Chauca CB. Deformidades torsionales de los miembros inferiores y la alteración delequilibrio dinámico en niños de 4 a 7 años: distrito del Callao, 2008 [tesis doctoral]. Lima- Perú: Universidad Nacional Mayor de San Marcos; 2008.

43. Villalobos J, Parodi A, Gonzalez R. estandarización de valores cinemáticos en niños sanos. Rev Mex Med Fis Rehab. 2005; 17: 47-53.

44. Mickle KJ, Cliff DP, Munro BJ, Okely AD, Steele JR. relationship between plantar pressures, physical activity and sedentariness among preschool children. L Sci Med Sport. 2011; 14(1): 36-41.

45. Bosch K, Nagel A, Weigend L, Rosenbaum D. From "first" to "last" steps in life-pressure patterns of three generations. Clin Biomech. 2009; 24(8): 676-681.

46. Echarri JJ, Forriol F. Desarrollo de la morfología de la huella plntar en niños congoleños y su relación con el uso del calzado. Rev Ortop Traumatol. 2003; 47: 395-399.

47. Zurita F. Screening y prevalencia de las alteraciones raquídeas (escoliosis e hipercifosis) en una población escolar de 8 a 12 años de Granada y provincia [tesis doctoral]. Granada: Universidad de Granada; 2007.

48. Nicasio J, Dáz F, Rivera A. Evaluación musculoesquelética en niños escolares que practican fútbol. Rev Mex Ortop Traumatol. 1994; 8(4); 191-105.

49. Ferrer V, Santonja F, Contreras M, Martínez I, Martínez L, Carrión M, et al. Alteraciones en el aparato locomotor del joven deportista. En: Ferrer V, Martínez L, Santonja F, eds. Escolar: Medicina y Deporte. Albacete: Diputación de Albacete, 1996; 369-378.

50. Baar A, Ibañez A, Gana N. Pie plano flexible: ¿Qué y porqué tratar? Rev Chil Pediatr. 2006; 77(4): 350-354.

51. Revenga-Giertych C, Bulo-Concellón MP. El pie plano valgo: evolución de la huella plantar y factores relacionados. Rev Ortop Trumatol. 2005; 49:271-280.

52. Espinoza-Navarro O, Valle S, Berrios G, Horta J, Rodriguez H, Rodriguez M. Prevalencia de alteraciones posturales en niños de Arica-Chile. Efectos de un programa de mejoramiento de la postura. Int J Morphol. 2009; 27(1): 25-30.

53. Molano NJ. Características posturales de los niños de la escuela "Jose Maria Obando" de la ciudad de Popayán. EFDeportes [revista en internet] 2004 [consultado el 2 de abril de 2013]; Año 10(70): [aproximadamente 9 páginas]. Disponible en: http//www.efdeportes.com.

54. Ballestero R. Efectividad del abordaje fisioterápico global a través del método G.D.S en el pie plano flexible infantil. Reduca: serias trabajos fin de máster. 2010; 2(1): 96-114.

55. Yabar LF, García DO, Cóndor C, Villavicencia E. Estudio para el diseño de un sistema baropodométrico electrónico. En: XVI Encuentro Científico Internacional de Verano ECI2009Epónimo "Pedro Salcedo Esparza". Universidad Tenológica del Perú. 2001: 1-4.

56. Carlier B. Hábitos podológicos en una población universitaria. Reduca: serie congresos alumnos. 2001; 3(13): 5-6.

57. AAOS [sede web]. Illinois: American Academy of Orthopaedic Surgeons; 2009 [consultado el 5 de abril de 2013]. Don't rely on stated shoe size. Children at risk for foot deformity due to wrong size shoes [1 pantalla]. Disponible en: http://www.aaos.org/news/aaosnow/mar09/clinical5.asp

58. Arocena JI, Fernández MA,. Dolor musculo-esquelético en pacientes pediátricos. Prevalencia y etiología en atención primaria. An Esp Pediatr. 1998-, 48: 381-384.

59. García CG. Exploración ortopédica del niño con dolor de espalda. En: AEPap ed. Curso de actualización pediátrica 2010. Madrid: Exlibris Ediciones; 2010. pp. 259-264.

60. Evans AM, Scutter SD. Prevalence of "growing pains" in Young children. J Pediatr. 2004; 145(2): 255-258.

61. Sass P, Hassan G. Lower extremity abnormalities in Children. Am Fam Physycian. 2003; 68(3): 461468.

62. Benedetti MG, Ceccareli F, Berti L, Luciani D, Catani F, Boshi M, et al. Diagnosis of flexible flat foot in children: a systematic clinical approach. Ortiopedics. 2011; 34(2): 94.

63. Kirby K. Biomechanics of the normal and abnormal foot. J Am Podiatr Med Assoc. 2000; 90(1): 30-34. 